\title{
Breast cancer in multiple endocrine neoplasia type 1 (MEN1)
}

\author{
Seong Keat Cheah', Chad Ramese Bisambar1, Deborah Pitfield', Olivier Giger², \\ Rogier ten Hoopen², Jose-Ezequiel Martin³, Graeme R Clark³, Soo-Mi Park ${ }^{3}$, \\ Craig Parkinson ${ }^{4}$, Benjamin G Challis ${ }^{1}$ and Ruth T Casey ${ }^{1,3}$
}

${ }^{1}$ Endocrinology, 2 Pathology, ${ }^{3}$ Medical Genetics, Addenbrookes Hospital, Cambridge University Hospitals NHS Foundation Trust, Cambridge, Cambridgeshire, UK, and ${ }^{4}$ Endocrinology, East Suffolk and North Essex NHS

Foundation Trust, Colchester, Essex, UK
Correspondence

should be addressed

to B G Challis or R T Casey

Email

bc340@medschl.cam.ac.uk or rc674@medschl.cam.ac.uk

\section{Summary}

A 38-year-old female was identified as carrying a heterozygous pathogenic MEN1 variant (c.1304delG) through predictive genetic testing, following a diagnosis of familial hyperparathyroidism. Routine screening for parathyroid and pituitary disease was negative. However, cross-sectional imaging by CT revealed a $41 \mathrm{~mm}$ pancreatic tail mass. Biopsy via endoscopic ultrasound confirmed the lesion to be a well-differentiated (grade 1) pancreatic neuroendocrine tumour (pNET) with MIB1<1\%. Biochemically, hyperinsulinaemic hypoglycaemia was confirmed following an overnight fast, which was subsequently managed by diet alone prior to definitive surgery. Pre-operative work-up with octreotide SPECT CT demonstrated avid tracer uptake in the pancreatic lesion and, unexpectedly, a focal area of uptake in the left breast. Further investigation, and subsequent mastectomy, confirmed ductal carcinoma in situ pT2 $(23 \mathrm{~mm})$ grade 1, N0 (ER positive; HER2 negative). Following mastectomy, our patient underwent a successful distal pancreatectomy to resect the PNET. Loss of heterozygosity ( $\mathrm{LOH}$ ) at the MEN1 locus was found in both the breast tumour and PNET, thereby in keeping with a 'two-hit' hypothesis of oncogenesis, a suggestive but non-definitive clue for causation. To obtain further support for a causative relationship between MEN1 and breast cancer, we undertook a detailed review of the published literature which overall supports the notion that breast cancer is a MEN1-related malignancy that presents at a younger age and histologically, is typically of ductal subtype. Currently, clinical guidance regarding breast cancer surveillance in MEN1 does not exist and further research is required to establish a clinical and cost-effective surveillance strategy).

\section{Learning points:}

- We describe a case of pNET and breast cancer diagnosed at a young age of 38 years in a patient who is heterozygous for a pathogenic MEN1 variant. Loss of the wild-type allele was seen in both breast tissue and pNET specimen.

- Breast cancer may be an under-recognised MEN1-associated malignancy that presents at a younger age than in the general population with a relative risk of 2-3.

- Further research is required to determine the cost-effectiveness of breast cancer surveillance approach at a younger age in MEN1 patients relative to the general population . 


\section{Background}

MEN1 is a rare autosomal dominant condition resulting from inactivating mutations of the MEN1 gene, which normally encodes for menin, a 610-amino acid protein responsible for tumour suppression (1). MEN1 is associated with a spectrum of endocrinological disorders that classically includes primary hyperparathyroidism (PHP), duodenopancreatic neuroendocrine tumours (pNETs) and pituitary adenomas. Among this triad, primary hyperparathyroidism is the most common clinical manifestation of MEN1, with almost complete penetrance by 50 years of age (2). pNETs have a lifetime penetrance of $30-75 \%$, while pituitary adenomas have a penetrance of $30-40 \%$ (3). Given the prevalence of these conditions and the importance of their early diagnosis and timely intervention, recommendations regarding their respective surveillance strategies are the focus of the most recent clinical practice guidance for MEN1 (3). However, it is increasingly recognised that the spectrum of MEN1related tumours also includes a number of less prevalent tumours including small bowel neuroendocrine tumours, adrenal tumours (adenomas and adenocarcinomas), lipomas, angiofibromas, collagenomas and meningiomas. Clearly, a heightened awareness of these associations and an early diagnosis of these conditions are critical for preventing morbidity and/or premature mortality in this patient group (3). In addition to these rare tumours, several studies have also reported an increased prevalence of breast cancer in MEN1 compared with the general population $(4,5)$. However, whether breast cancer is truly a MEN1-related malignancy has been contested $(5,6)$. Here, we report a case of breast cancer co-existing with an insulin-secreting pNET in a young female with MEN1. We have demonstrated MEN1 LOH in both tumours, which, supported by a review of the published literature, provides further evidence that breast cancer is a MEN1-related malignancy that should be considered for inclusion tumour surveillance strategies for MEN1.

\section{Case presentation}

A 38-year-old female was identified as being heterozygous for a MEN1 pathogenic variant (c.1304delG) through predictive genetic testing, following a diagnosis of familial hyperparathyroidism in her mother and maternal aunt, who harboured the same mutation. Her maternal grandparents were deceased; however, her grandmother was known to have breast cancer and died at age 62 , and her grandfather had a brain tumour diagnosed at age 41 .
Our patient has two healthy sons (aged 5 and 7) neither of which has been tested for MEN1 (Fig. 1).

In addition to a recent diagnosis of primary hyperparathyroidism, our patient's medical history was significant for anxiety and panic attacks for which she took sertraline.

During her first consultation in the neuroendocrine tumour outpatient clinic following genetic confirmation of MEN1, a focused systems review revealed clinical features suggestive of hypoglycaemia that included episodic sweating, confusion and reduced consciousness, which quickly reversed the following ingestion of carbohydrate and were precipitated by missed meals or physical activity.

Clinical examination revealed a body weight of $55 \mathrm{~kg}$ and BMI of $22 \mathrm{~kg} / \mathrm{m}^{2}$. Our patient did not manifest any overt clinical features of pituitary hormone dysfunction. She was clinically euthyroid and her visual fields were unremarkable.

\section{Investigation}

Biochemical screening for hyperparathyroidism and pituitary disease was unremarkable during the initial clinic visits. However, a cross-sectional imaging by CT revealed a $41 \mathrm{~mm}$ pancreatic tail mass (Fig. 2A). Subsequent fine needle aspiration and cytological examination during an endoscopic ultrasound confirmed the diagnosis of a well-differentiated (grade 1) pancreatic neuroendocrine tumour (pNET) with MIB1<1\%.

Given the clinical history suggestive of hypoglycaemia, the patient underwent an overnight fasting test. After $11 \mathrm{~h}$ of fasting, she developed hypoglycaemia at a plasma glucose level of $2.2 \mathrm{mmol} / \mathrm{L}$, with paired insulin measurement at $51 \mathrm{pmol} / \mathrm{L}$ and C-peptide of $801 \mathrm{pmol} / \mathrm{L}$, confirming hyperinsulinaemic hypoglycaemia. This was managed effectively by diet modification prior to definitive surgery.

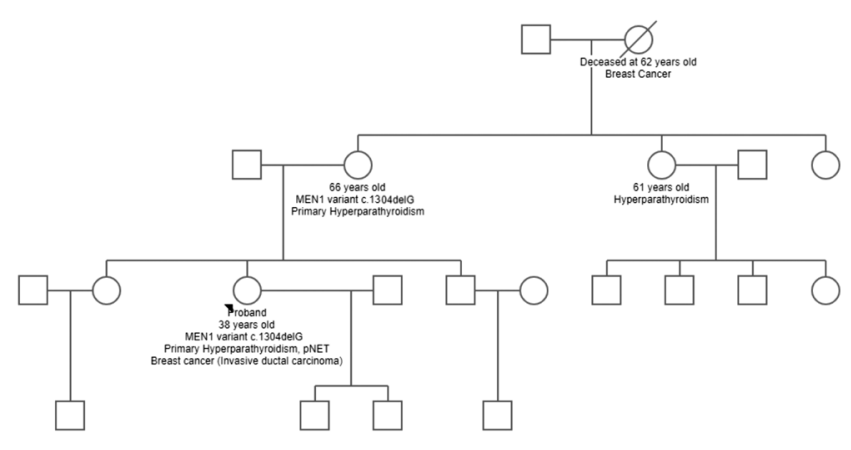

Figure 1

Family tree with background familial hyperparathyroidism. 

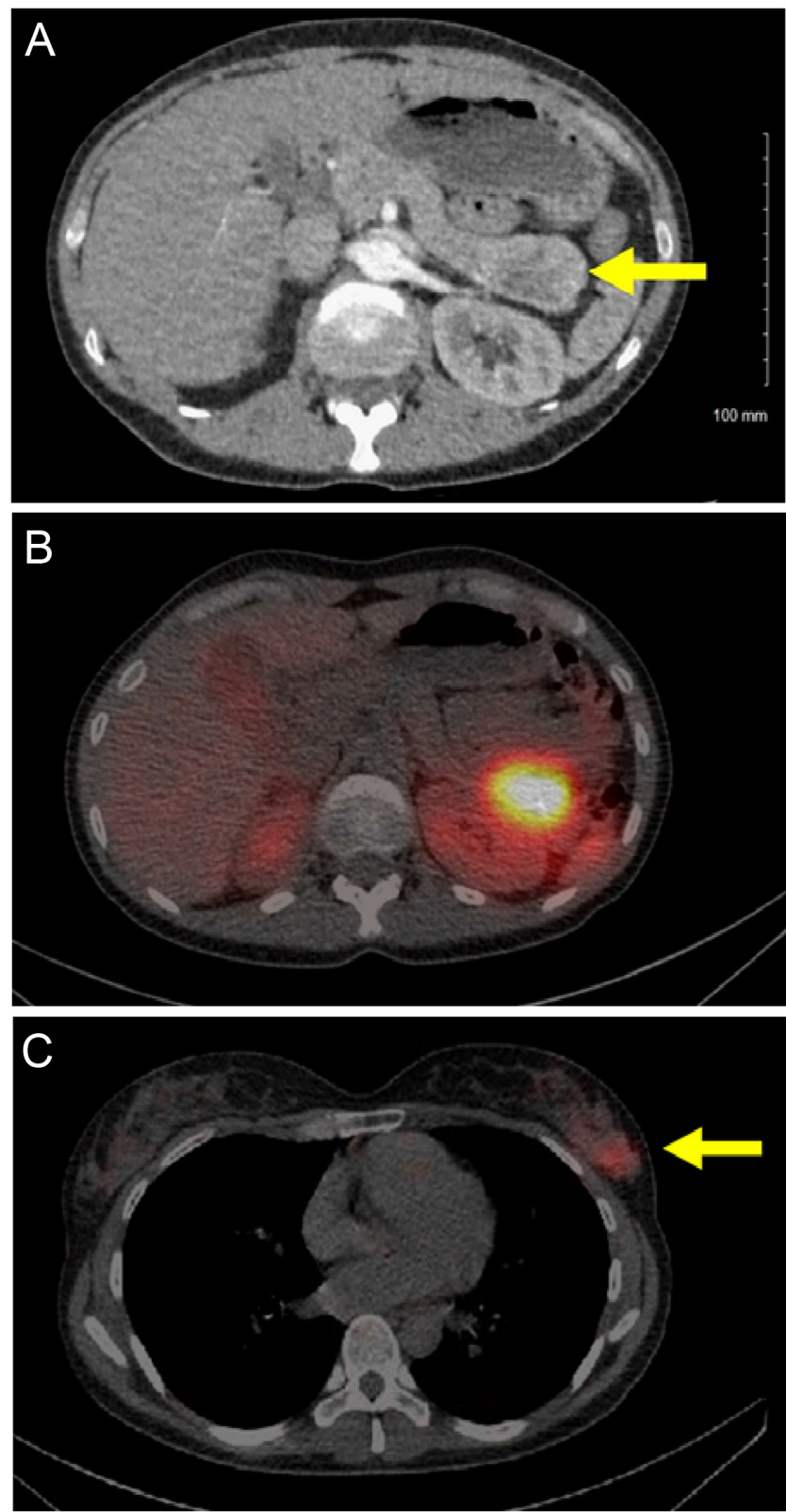

Figure 2

(A) Cross-sectional CT revealed a $41 \times 29 \mathrm{~mm}$ heterogeneous mass in the tail of the pancreas. (B) Octreotide SPECT CT showing avid uptake in the pancreatic mass. (C) Octreotide SPECT CT showing avid uptake in the left breast (lower panel, yellow arrow).

In preparation for surgery, a pre-operative Octreotide SPECT CT was performed which demonstrated avid tracer uptake in the pancreatic lesion (Fig. 2B). Unexpectedly, however, an area of focal uptake was also seen in the left breast (Fig. 2C), and following biopsy, the diagnosis of breast carcinoma was made.

\section{Treatment}

Following clinical review, and multidisciplinary discussion involving the patient, the decision was made to first undertake a left mastectomy followed by distal pancreatectomy. The rationale for this decision reflected the increased potential of post-operative complications following a distal pancreatectomy and prolonged recovery period which may have delayed the planned mastectomy which was deemed to be the clinical priority. Reassuringly, her blood glucose levels were adequately supported by diet alone and closely monitored, which mitigated against the risk of severe hypoglycaemia whilst waiting for pNET resection. Therefore, the patient proceeded to an uncomplicated left mastectomy.

Subsequent histological examination confirmed a grade 1 ductal carcinoma in situ of $23 \mathrm{~mm}$ (pT2), with no metastasis to lymph nodes (N0) or distant organs (M0). The patient was commenced on tamoxifen in light of oestrogen receptor (ER) immunopositivity. The tumour was not immunopositive for progesterone receptor (PR) expression or Herceptin-2 receptor (HER2) expression.

Eight weeks following mastectomy, our patient underwent successful and uncomplicated distal pancreatectomy and splenectomy. Multifocal grade 1 welldifferentiated neuroendocrine tumours were identified histologically. They were of varying sizes with diameters of $35,20,14$, and $8 \mathrm{~mm}$, respectively. There was no local lymphatic spread and the tumours were focally less than $1 \mathrm{~mm}$ from the posterior margin. The MIB- 1 index was $<1 \%$. Immunohistochemical staining for insulin was described as patchy. According to the ENET (European Neuroendocrine Tumour Society) staging criteria, the pNET was staged at pT2, N0, M0, recognising that breast cancer is a histologically distinct entity (7).

\section{Outcome and follow-up}

Both surgical procedures were successful in achieving disease clearance and the patient has remained euglycaemic. The well-differentiated nature of her lowgrade pNET, the absence of lymph node spread on both disease sites and pre-operative octreotide SPECT CT which did not show additional uptake elsewhere, is favourable for her prognosis. However, being a MEN1 carrier, disease recurrence in the remaining pancreas remnant is possible and the patient will continue to receive lifelong biochemical and radiological surveillance. 
The synchronous identification of breast cancer prompted us to investigate the genetic makeup of the breast lesion and its relation to the MEN1 carrier state. DNA sequencing of the MEN1 locus was performed using a germline sample, from the patient, breast tumour tissue and pNET specimen after informed consent was obtained (REC 14/EE/105). Tumour microdissection of the relevant paraffin-embedded tumour samples was performed. Tissue slides were deparaffinised and DNA was extracted using the Covaris Adaptive Focused Acoustics ${ }^{\mathrm{TM}}$ (AFA) based DNA extraction and purification technique. DNA was sequenced using a modified AmpliSeq cancer hotspot panel, containing 142 Cancer Hotspot Panel v2 (catalogue number 4475346) by the staff at the Stratified Medicine Core Laboratory within the Department of Medical Genetics, Cambridge University. Tumour DNA from the breast tumour was also sequenced using SeqCap EZ HyperCap workflow (Roche Sequencing Solutions, Inc.), utilising a targeted sequencing method containing a 350 gene panel in collaboration with colleagues from the Cancer and Molecular Diagnostics Laboratory in Cancer Research UK Cambridge. Variant filtering was performed on variant calling files (VCF). Variants were removed if the variant allele frequency was $<5 \%$. Synonymous variants were removed as presumed not to be pathogenic. Benign variants, variants of uncertain significance and those variants that had coverage of less than two standard deviations below the mean coverage were also removed.

Biallelic loss in the breast tumour and pNET at the MEN1 locus were suggested by the finding of higher reads of the mutant allele identified in the breast tumour (variant allele frequency 73\%) and the pNET (variant allele frequency 90\%) compared with the germline (variant allele frequency 51\%). Somatic pathogenic truncating variants in BRCA2 and TP53 were also identified in the breast tumour in our patient but the variant allele frequency was $<10 \%$ for both variants, suggesting that these variants were sub-clonal mutations rather than a primary driver mutation. No further pathogenic or likely pathogenic driver somatic variants were identified on either of the two gene panels utilised for sequencing of the breast tumour.

\section{Reported cases and series}

We sought to correlate the clinical, histological and genetic characteristics of our case to other reported cases of MEN1 related carcinoma and performed a literature search using PubMed and Google Scholar. MeSH search terms applied in PubMed included 'Breast Neoplasms' and 'Multiple Endocrine Neoplasia Type 1'. Search terms applied in Google Scholar were 'breast', 'cancer', 'carcinoma', 'adenocarcinoma', 'MEN', 'MEN1' and 'multiple endocrine neoplasia type 1'. Features of interest to us were the age of breast cancer diagnosis, histological type, staging, hormone receptor expression, LOH status, and MEN1 genetic variant and these are listed in Table 1. Due to the relative rarity of the condition, we have included abstracts, case reports and reported series of varying quality, and we accept that some of the features are variably reported depending on the availability of local genetic services and the nature of the reporting literature.

\section{Discussion}

Menin, a protein coded by MEN1 is a ubiquitously expressed nuclear protein that has no intrinsic enzymatic activity, but it regulates transcription either as a co-activator or co-repressor. Dreijerink et al. have described menin's role in regulating antiproliferative genes in mammary progenitor cells, which may lead to oncogenesis (8).

\section{Features of reported MEN1 related breast cancer}

Despite the variability in reporting, the majority of breast cancer in MEN1 patients were diagnosed at a relatively younger age and the most common histological subtype was ductal carcinoma with variablity of ER/PR and HER2 expression (Table 1). They are usually diagnosed at early stages and no cases of metastatic disease have been reported thus far.

The largest reported series was published by the Dutch MEN1 study group which includes over $90 \%$ of MEN1 Dutch population with 20 years of mean follow-up duration (2). From this cohort, $73 \%$ of female MEN1 patients who had breast cancer reported no family history of breast malignancy. Relative to control group, no significant differences were found for the presence of breast cancer risk factors including menarche age, menopause age, age at first delivery, practice of breast feeding, use of oral contraceptive pills, obesity, smoking and alcohol consumption. The same group reported that from a cohort of 190 MEN1 females, 12 cases (6.3\%) of breast cancer were identified, resulting in a relative risk of invasive breast cancer of 2.83 consistent with similar findings in three other independent validation cohorts in US ( $n=68$, RR: $2.40, P=0.11)$, Tasmania $(n=71$, RR: 2.31, $\mathrm{P}=0.22)$ and France $(n=536, \mathrm{RR}: 2.33, P=0.03)$ (5). However, the comparisons were not statistically 
Table 1 Reported cases of breast cancer in MEN1 patients.

\begin{tabular}{|c|c|c|c|c|c|c|c|c|c|}
\hline No. & Age & Histology & TNM staging & ER & PR & HER2 & LOH & MEN1 variant & Reference \\
\hline 1 & 44 & Scirrhous & $\mathrm{T} 1 \mathrm{~N} \times \mathrm{Mx}$ & $\mathrm{NA}$ & $\mathrm{NA}$ & $\mathrm{NA}$ & + & c. $1621 \mathrm{G}>\mathrm{A}$ & (18) \\
\hline 2 & 45 & Ductal & $\mathrm{T} 2 \mathrm{~N} \times \mathrm{M} 0$ & + & + & - & NA & c.196_200dupAGCCC (frameshift) & (19) \\
\hline 3 & 55 & Ductal & $\mathrm{T} 1 \mathrm{~N} 1 \mathrm{M} 0$ & + & - & - & - & NA & (5) \\
\hline 4 & 38 & Ductal & T3N1M0 & + & + & + & - & NA & (5) \\
\hline 5 & 44 & Ductal & T1N0M0 & - & - & -- & - & NA & (5) \\
\hline 6 & 61 & Ductal & T1N1M0 & + & - & + & - & NA & (5) \\
\hline 7 & 52 & Lobular & T1NOMO & + & + & - & - & NA & (5) \\
\hline 8 & 53 & Ductal & T1NOMO & + & + & - & + & NA & (5) \\
\hline 9 & 45 & Micropapillary & T1N1M0 & + & - & - & + & NA & (5) \\
\hline 10 & 42 & Ductal (bilateral) & T1N0M0 & - & + & & + & NA & (5) \\
\hline 11 & 33 & Ductal & T1N1M0 & + & + & + & - & NA & (5) \\
\hline 12 & 46 & Ductal & T1N0M0 & + & + & - & NA & NA & (5) \\
\hline 13 & 36 & Ductal & NA & + & + & + & NA & c.1649insC & (20) \\
\hline 14 & 41 & Ductal & T1NOMO & - & - & - & NA & NA & $(21)$ \\
\hline 15 & 56 & Ductal & NA & NA & NA & NA & NA & c.628_631delACAG & $(22)$ \\
\hline 16 & 48 & Ductal & T1 N0M0 & + & + & - & NA & c.1365+1_1365+11 del, GTGAGGGACAG & $(23)$ \\
\hline 17 & 38 & Ductal & T2NOMO & + & + & - & + & c.1304delG & This case \\
\hline
\end{tabular}

A plus sign indicates positivity, and a minus sign negativity.

HER2, human EGF receptor 2; NA, not available.

significant between the later two cohorts, presumed to be due to the smaller sample sizes. Additionally, comparisons may be confounded by variable sample sizes and duration of follow-up between cohorts (6).

The Dutch cohort had early onset breast cancer diagnosed at a young median age of $48.0 \pm 8.8$ years, as compared to the age of 60-65 in general population (5). In relation to this, our patient was diagnosed with breast cancer at 38 years of age.

However, MEN1 variants were not found to be significantly associated with breast cancer when a panel of 34 putative susceptibility genes was tested in a populationbased study of 48826 women with breast cancer and 50 703 controls. In this study, a truncating MEN1 variant was identified in two women with breast cancer and five controls with an odds ratio of 0.37 (95\% CI: 0.07-1.97, $P=0.24$ ), while a missense variant in MEN1 was identified in 109 women with breast cancer and 130 controls, with an odds ratio of 0.86 (95\% CI: $0.66-1.12, P=0.25)(9)$.

\section{LOH analysis}

$\mathrm{LOH}$ analysis is important to recognise if there is a presence of biallelic loss of a specific tumour suppressor gene. Possession of a single loss-of-function mutant MEN1 copy in the germline DNA will predispose to tumour development. In line with a 'two-hit hypothesis', if subsequent somatic (acquired) loss-of-function of the second allele in a cell occurs, it will then result in MEN1related tumour (10). Concomitant loss (mutation) of both copies of allele is termed LOH, a feature suggestive yet not conclusive of causation. In our patient, tumour sequencing has suggested biallelic loss in the breast tissue and pNET specimen, with a mutant allele frequency of 73 and 91\%, respectively. Although, it should be noted that formal analysis for loss of heterozygosity was not performed and copy number analysis was not possible due to the nature of the sequencing performed (targeted gene panel sequencing).

Reports of $\mathrm{LOH}$ analysis in the reported cases of breast cancer in MEN1 have been varied (Table 1). This may reflect the variation in access to specialist genetic laboratory testing from one institution to another. Moreover, it appears that $\mathrm{LOH}$ at breast tissue level may be of low yield as suggested in the largest series from the Dutch group as only three out of nine breast specimens were demonstrated to have $\mathrm{LOH}$ (5).

Presence of tumour $\mathrm{LOH}$ is limited in proving causation as it can be a common secondary event in tumours. For instance, deletions or rearrangement of the long arm of chromosome 11 is commonly seen in other tumours. Therefore, the $\mathrm{LOH}$ analysis technique is important where the sequencing of MEN1 in tumour DNA and a dosage assay (MLPA) of MEN1 alone are probably 
less fallible than dosage analysis of a larger section of chromosome 11 containing the MEN1 gene locus $(6,11)$.

\section{The role of BRCA}

BRCA1 and BRCA2 are the main driver genes for breast carcinogenesis. There were reports of MEN1 females who also had mutations of BRCA1 (12) and BRCA2 (13). These cases did not present with breast cancer when they were reported. The Dutch cohort of MEN1 females with breast cancer was derived from ten different families, and of these, only two satisfied the Dutch national criteria for BRCA1/2 genetic screening and were proven negative (4). Our patient was not tested for BRCA1 and BRCA2 as her family history does not fulfil current NHS criteria for testing.

Somatic truncating BRCA2 and TP53 mutations were also identified at low variant allele frequency $(<10 \%)$ in the breast tumour in our patient, and, therefore, they were identified as sub-clonal mutations rather than potential driver mutations. The interpretation of our sequencing results is limited by our inability to undertake genetic studies in deceased relatives with a history of breast cancer (Fig. 1) and as a targeted gene panel was employed rather than a whole exome sequencing approach, we cannot exclude the possibility of other causative genetic mutations in the breast tumour.

\section{Future research needs and clinical practice}

In the UK, women aged 50-70 years are invited to breast screening programs every 3 years (14). This strategy may miss the opportunity of diagnosing breast cancer early in MEN1 patients who were reported to present younger. Early detection of young-onset malignancy may potentially be curative prior to disease progression. However, with inadequate evidence to ascertain association at the moment, it is not immediately obvious if enhanced breast cancer surveillance is justifiable. The prognostic significance of MEN1 mutation upon breast cancer diagnosis is also unclear to inform surveillance and management.

The existing clinical practice guideline for MEN1 was published in 2012 before the evidence for potential breast cancer association started to emerge (3). Therefore, the guideline does not include specific recommendations with regards to breast cancer surveillance and management in MEN1. However, considering the younger age of diagnosis of breast cancer in MEN1 identified from data obtained from the Dutch MEN1 cohort, the authors have suggested that biennial breast cancer screening from age 40 may be justifiable in MEN1 patients (15). This is in line with simulation modelling suggesting that screening breast cancer annually in women above 40 years of age who are at two- to four-fold increase risk of breast cancer had similar or more favourable harm-to-benefit ratios as biennial screening of 50- to 74-year-old women at average risk (16). This appears to be applicable for the MEN1 group, for whom the breast cancer risk was estimated to be almost three-fold higher by the Dutch group (5).

The evidence to date suggesting that the risk of breast cancer in women who are heterozygous for MEN1 pathogenic variants is two- to three-fold greater than in women in the general population with an earlier onset would fulfil criteria for moderate risk of breast cancer under current NHS breast screening guidelines (17). The recommended screening for this consists of annual mammography between the age of 40 and 50 years followed by the routine NHS breast screening programme from the age of 50 years.

\section{Declaration of interest}

BGC is an employee of AstraZeneca. Other authors have no conflict of interest.

\section{Funding}

This research did not receive any specific grant from any funding agency in the public, commercial or not-for-profit sector.

\section{Patient consent}

Written informed consent has been obtained from the patient for publication of the case report and accompanying images.

\section{Author contribution statement}

Seong Keat Cheah is an Endocrinology specialty trainee who wrote this article and was involved in the endocrinological care of this patient along with Chad Bisambar (Endocrinology clinical fellow) and Deborah Pitfield (Endocrinology specialist nurse). Olivier Giger and Rogier ten Hoopen analysed and reported the pathology specimen. Craig Parkinson, Benjamin $\mathrm{G}$ Challis and Ruth T Casey are the named consultant endocrinologists who were responsible for patient's endocrinological management. Ruth T Casey being the senior author had also performed DNA sequencing reported in this article, with technical support and guidance from colleagues from Medical Genetics, Jose Ezequiel Martin, Graeme R Clark and Soo-Mi Park. Tumour microdissection of the relevant paraffin-embedded tumour samples was performed by colleagues in the Department of Haematology and Oncology diagnostic services, Cambridge University Hospital.

\section{References}

1 Chandrasekharappa SC, Guru SC, Manickam P, Olufemi SE, Collins FS, Emmert-Buck MR, Debelenko LV, Zhuang Z, Lubensky IA, 
Liotta LA, et al. Positional cloning of the gene for multiple endocrine neoplasia-type 1. Science 1997276 404-407. (https://doi. org/10.1126/science.276.5311.404)

2 Rizzoli R, Green J \& Marx SJ. Primary hyperparathyroidism in familial multiple endocrine neoplasia type I. Long-term follow-up of serum calcium levels after parathyroidectomy. American Journal of Medicine 198578 467-474. (https://doi.org/10.1016/00029343(85)90340-7)

3 Thakker RV, Newey PJ, Walls GV, Bilezikian J, Dralle H, Ebeling PR, Melmed S, Sakurai A, Tonelli F, Brandi ML, et al. Clinical practice guidelines for multiple endocrine neoplasia type 1 (MEN1). Journal of Clinical Endocrinology and Metabolism 201297 2990-3011. (https:// doi.org/10.1210/jc.2012-1230)

4 van Leeuwaarde RS, Dreijerink KM, Ausems MG, Beijers HJ, Dekkers OM, de Herder WW, van der Horst-Schrivers AN, Drent ML, Bisschop PH, Havekes B, et al. MEN1-dependent breast cancer: indication for early screening? Results from the Dutch MEN1 study group. Journal of Clinical Endocrinology and Metabolism 2017102 2083-2090. (https://doi.org/10.1210/jc.2016-3690)

5 Dreijerink KMA, Goudet P, Burgess JR, Valk GD \& International Breast Cancer in MEN1 Study Group. Breast-cancer predisposition in multiple endocrine neoplasia type 1. New England Journal of Medicine 2014371 583-584. (https://doi.org/10.1056/NEJMc1406028)

6 Brennan P. Breast cancer risk in MEN1 - a cancer genetics perspective. Clinical Endocrinology 201582 327-229. (https://doi.org/10.1111/ cen.12614)

7 Rindi G, Klöppel G, Alhman H, Caplin M, Couvelard A, de Herder WW, Erikssson B, Falchetti A, Falconi M, Komminoth P, et al. TNM staging of foregut (neuro)endocrine tumors: a consensus proposal including a grading system. In Virchows Archiv $2006 \mathbf{4 4 9}$ 395-401. (https://doi.org/10.1007/s00428-006-0250-1)

8 Dreijerink KMA, Groner AC, Vos ESM, Font-Tello A, Gu L, Chi D, Reyes J, Cook J, Lim E, Lin CY, et al. Enhancer-mediated oncogenic function of the menin tumor suppressor in breast cancer. Cell Reports 201718 2359-2372. (https://doi.org/10.1016/j.celrep.2017.02.025)

9 Breast Cancer Association Consortium, Dorling L, Carvalho S, Allen J, González-Neira A, Luccarini C, Wahlström C, Pooley KA, Parsons MT, Fortuno C, et al. Breast cancer risk genes association analysis in more than 113,000 women. New England Journal of Medicine 2021384 428-439. (https://doi.org/10.1056/ NEJMoa1913948)

10 Pannett AAJ \& Thakker RV. Somatic mutations in MEN Type 1 tumors, consistent with the Knudson "two-hit" hypothesis. Journal of Clinical Endocrinology and Metabolism 200186 4371-4374. (https:// doi.org/10.1210/jcem.86.9.7844)

11 Dreijerink KMA \& Valk GD. Reply to: breast cancer risk in MEN1 - a cancer genetics perspective. Clinical Endocrinology: Blackwell Publishing Ltd 201583 141. (https://doi.org/10.1111/cen.12679)

12 Papi L, Palli D, Masi L, Putignano AL, Congregati C, Zanna I, Marini F, Giusti F, Luzi E, Tonelli F, et al. Germline mutations in MEN1 and BRCA1 genes in a woman with familial multiple endocrine neoplasia type 1 and inherited breast-ovarian cancer syndromes: a case report. Cancer Genetics and Cytogenetics 2009195 75-79. (https://doi.org/10.1016/j.cancergencyto.2009.06.019)

13 Ghataorhe P, Kurian AW, Pickart A, Trapane P, Norton JA, Kingham K \& Ford JM. A carrier of both MEN1 and BRCA2 mutations: case report and review of the literature. Cancer Genetics and Cytogenetics 2007179 89-92. (https://doi.org/10.1016/j. cancergencyto.2007.08.009)

14 Independent UK Panel on Breast Cancer Screening. The benefits and harms of breast cancer screening: an independent review. Lancet 2012380 1778-1786. (https://doi.org/10.1016/S01406736(12)61611-0)

15 van Leeuwaarde RS, de Laat JM, Pieterman CRC, Dreijerink K, Vriens MR \& Valk GD. The future: medical advances in MEN1 therapeutic approaches and management strategies. EndocrineRelated Cancer 201724 T179-T193. (https://doi.org/10.1530/ERC-170225)

16 Mandelblatt JS, Stout NK, Schechter CB, van den Broek JJ, Miglioretti DL, Krapcho M, Trentham-Dietz A, Munoz D, Lee SJ, Berry DA, et al. Collaborative modeling of the benefits and harms associated with different U.S. Breast cancer screening strategies. Annals of Internal Medicine 2016164 215-225. (https://doi. org/10.7326/M15-1536)

17 National Institute for Health and Care Excellence. Familial breast cancer: classification, care and managing breast cancer and related risks in people with a family history of breast cancer NICE, 2013. (available at: https://www.nice.org.uk/guidance/cg164). (updated 2019 Nov).

18 Honda M, Tsukada T, Horiuchi T, Tanaka R, Yamaguchi K, Obara T, Miyakawa H, Yamaji T \& Ishibashi M. Primary hyperparathyroidism associatiated with aldosterone-producing adrenocortical adenoma and breast cancer: relation to MEN1 gene. Internal Medicine 200443 310-314. (https://doi.org/10.2169/internalmedicine.43.310)

19 Jeong YJ, Oh HK \& Bong JG. Multiple endocrine neoplasia type 1 associated with breast cancer: a case report and review of the literature. Oncology Letters 20148 230-234. (https://doi.org/10.3892/ ol.2014.2144)

20 Elezovic V, Petakov M, Macut D, Ognjanovic S, Isailovic T, Milicevic I, Popovic B, Antic IB, Bogavac T, Ilic D, et al. Breast cancer in multiple endocrine neoplasia type 1. Endocrine Abstracts 201435 P299. (https://doi.org/10.1530/endoabs.35.P299)

21 Herranz-Antolín S, Gil-García S \& Álvarez-de Frutos V. Multiple endocrine neoplasia type 1 and breast cancer. An association to consider. Endocrinologia, Diabetes y Nutricion 201865 468-469. (https://doi.org/10.1016/j.endinu.2018.05.008)

22 Larouche V, Akirov A, Thain E, Kim RH \& Ezzat S. Co-occurrence of breast cancer and neuroendocrine tumours: new genetic insights beyond multiple endocrine neoplasia syndromes. Endocrinology, Diabetes and Metabolism 20192 e00092. (https://doi.org/10.1002/ edm2.92)

23 Kim SH \& Park JH. Adrenal incidentaloma, breast cancer and unrecognized multiple endocrine neoplasia type. Acta Endocrinologica 201915 513-517. (https://doi.org/10.4183/aeb.2019.513)
Received in final form 22 March 2021

Accepted 13 April 2021 